\title{
T-complex protein 1 subunit zeta-2 (CCT6B) deficiency induces murine teratospermia
}

\author{
Peiyin Yang ${ }^{\text {Equal first author, } 1}$, Wenjing Tang ${ }^{\text {Equal first author, } 1}{ }^{1}$, Huiling Li ${ }^{1}$, Rong Hua ${ }^{1}$, Yan Yuan ${ }^{1}$, Yue Zhang ${ }^{1}$, Yunfei Zhu ${ }^{1}$, \\ Yiqiang Cui ${ }^{\text {Corresp., } 1}$, Jiahao Sha ${ }^{\text {Corresp. } 1}$ \\ ${ }^{1}$ Department of Histology and Embryology, Nanjing Medical University, Nanjing, Jiangsu, China \\ Corresponding Authors: Yiqiang Cui, Jiahao Sha \\ Email address: cuiyiqiang@126.com, shajh@njmu.edu.cn
}

Background: The CCT complex is an important mediator of microtubule assembly and intracellular protein folding. Owing to its high expression in spermatids, CCT knockdown can disrupt spermatogenesis. In the present report, we therefore evaluated the in vivo functionality of the testis-specific CCT complex component CCT6B using a murine knockout model system. Methods: A CRISPR/Cas9 approach was used to generate Cct6 $b^{-/}$mice, after which candidate gene expression in these animals was evaluated via qPCR and Western blotting. Testicular and epididymal phenotypes were assessed through histological and immunofluorescent staining assays, while a computer-assisted sperm analyzer was employed to assess semen quality. Results: $C c t 6 b^{-1}$ mice were successfully generated, and exhibited no differences in development, fertility, appearance, testis weight, or sperm counts relative to control littermates. In addition, no differences in spermatogenesis were detected when comparing $\mathrm{Cct} 6 b^{+/+}$and $C \operatorname{ct} 6 b^{-/}$testes. However, when progressive motility was analyzed, the ratio of normal sperm was significantly decreased in $\mathrm{Cct}_{6} \mathrm{~b}^{-/}$male mice, with nuclear base bending being the primary detected abnormality. In addition, slight decreases in Cct4 and Cct7 expression were detected.

Conclusion: These data indicated that CCT6B is an important regulator of murine spermatogenesis, with the loss of this protein resulting in CCT complex dysfunction, providing a foundation for further studies. 
2 T-complex protein 1 subunit zeta-2 (CCT6B) deficiency

3 induces murine teratospermia

4

5

6

8

Peiyin Yang\#, Wenjing Tang\#, Huiling Li, Rong Hua, Yan Yuan, Yue Zhang, Yunfei Zhu, Yiqiang Cui* and Jiahao Sha*

Department of Histology and Embryology, Nanjing Medical University, Nanjing, China

\#These authors contributed equally to this work

Corresponding Author. Email address: shajh@njmu.edu.cn (J.S.), cuiyiqiang@126.com (Y.C).

\section{Abstract}

Background: The CCT complex is an important mediator of microtubule assembly and intracellular protein folding. Owing to its high expression in spermatids, CCT knockdown can disrupt spermatogenesis. In the present report, we therefore evaluated the in vivo functionality of the testis-specific CCT complex component CCT6B using a murine knockout model system.

Methods: A CRISPR/Cas9 approach was used to generate $C c t 6 b^{-/}$mice, after which candidate gene expression in these animals was evaluated via qPCR and Western blotting. Testicular and epididymal phenotypes were assessed through histological and immunofluorescent staining assays, while a computer-assisted sperm analyzer was employed to assess semen quality.

Results: $C c t 6 b^{-/}$mice were successfully generated, and exhibited no differences in development, fertility, appearance, testis weight, or sperm counts relative to control littermates. In addition, no differences in spermatogenesis were detected when comparing $C c t 6 b^{+/+}$and $C c t 6 b^{-/}$testes. However, when progressive motility was analyzed, the ratio of normal sperm was significantly decreased in $\mathrm{Cct}_{\mathrm{C}} \mathrm{b}^{-/}$male mice, with nuclear base bending being the primary detected abnormality. In addition, slight decreases in $C c t 4$ and $C c t 7$ expression were detected.

Conclusion: These data indicated that CCT6B is an important regulator of murine spermatogenesis, with the loss of this protein resulting in CCT complex dysfunction, providing a foundation for further studies.

Keywords CCT6B, Spermatogenesis, Gene knockout, protein folding.

\section{Introduction}

Spermatogenesis is a complex process in which spermatogonial stem cells differentiate and develop to yield mature spermatozoa in an ordered process (Hess \& Renato de Franca 2008), which is composed of three primary steps. First, spermatogonial stem cells proliferate and differentiate to yield primary spermatocytes in what is known as the mitotic amplification phase or the premeiotic-phase. The spermatocytes then undergo a second round of meiotic division and develop 
40 into round haploid spermatids in the meiotic phase. In the final post-meiotic phase of 41 spermiogenesis, these round spermatids undergo morphological changes that lead to mature sperm 42 production (de Kretser et al. 1998). During this final phase, extensive remodeling occurs including 43 nuclear condensation, acrosome formation, flagellar development, and a loss of most cytoplasmic 44 material (Dias et al. 2016).

Sperm motility and structural integrity are closely linked to microtubule assembly and acrosome formation, and the disruption of these processes can have a severe adverse impact on fertility (Fouquet \& Kann 1994). Microtubules are composed primarily of tubulin, whereas the acrosome is made of actin. The folding of tubulin and actin is highly conserved in eukaryotic cells, being regulated by the multi-protein chaperonin-containing TCP-1 (CCT) complex (Dunn et al. 2001; Lopez et al. 2015; Valpuesta et al. 2002). This ATP-dependent chaperonin complex assists in the efficient folding of newly generated polypeptides (Broadley \& Hartl 2009; Rothman 1989), and is composed of two symmetrical rings containing 8 paralogous subunits encoded by TCP1, CCT2, CCT3, CCT4, CCT5, CCT6A or CCT6B, CCT7, and CCT8 (Kubota et al. 1995a; Spiess et al. 2004). The CCT1-8 subunits share a moderate degree of sequence identity $(\sim 30 \%)$, and orthologous proteins are highly similar across species, with $\sim 60 \%$ identity being shared between mammalian and yeast cells (Kubota et al. 1994; Kubota et al. 1995b). The sequence diversity of individual subunits is believed to provide appropriate substrate specificity through interactions with particular protein domains (Counts et al. 2017). Indeed, the CCT complex assists in the folding of roughly $15 \%$ of human proteins (Thulasiraman et al. 1999; Yam et al. 2008).

The CCT complex is a key mediator of protein folding in the context of spermatogenesis (Giuffrida et al. 2006; Souès et al. 2003; Zhu et al. 2006). In planarians, all eight CCT complex subunits have been shown to be critical for normal sperm development, and the relative levels of these proteins have the potential to influence the spermatogenic process (Counts et al. 2017). However, no knockout-based approaches to date have explored the role of CCT in murine spermatogenesis. There are two CCT6 homologs encoded in the human and murine genomes, with CCT6A being ubiquitously expressed whereas CCT6B is restricted to the testes (Kubota et al. 1997). The specific expression of CCT6B solely in the testes suggests it may be an important mediator of spermatogenesis, yet no data confirming this hypothesis have been published to date.

Genetic knockout models remain an essential tool for the in vivo assessment of the functions of specific genes, with CRISPR/Cas9 system-based approaches having been used on multiple occasions to target specific loci of interest in mammalian model systems (Mali et al. 2013; Shen et al. 2013; Wang et al. 2013). Indeed, knockout mice generated via this approach are commonly used for genetic studies (Castaneda et al. 2017; Hua et al. 2019; Jiang M 2014; Zhang et al. 2019). As such, we herein employed CRISPR/Cas9 approach to generate a 5-bp shift mutation in the fourth exon of the $C c t 6 b$ gene in $\mathrm{C} 57 \mathrm{~B} / 6 \mathrm{~J}$ mice, and we then used these animals to assess the reproductive impacts of CCT6B knockout. The results of these analyses suggest that $C c t 6 b$ is an important regulator of spermiogenesis.

\section{Materials \& Methods}


80

81

82

83

84

85

86

87

88

89

90

91

92

93

94

95

96

97

98

99

100

101

102

103

104

105

106

107

108

109

110

111

112

113

114

115

116

117

118

119

\section{Animals}

Animal care and treatment protocols were designed based upon the guidelines of the Institutional Animal Care and Use Committee (IACUC) of Nanjing Medical University, and all protocols described herein have received approval from the Animal Ethical and Welfare Committee (Approval No. IACUC-2009002-1).

All mice were obtained from and maintained under SPF conditions in the Laboratory Animal Center of Nanjing Medical University. Mice were housed in a climate-controlled facility $\left(20-22^{\circ} \mathrm{C}\right.$, $50-70 \%$ humidity, $12 \mathrm{~h}$ light/dark cycle) with free food and water access. All mice were treated humanely and all efforts were made to minimize suffering. When appropriate, mice were euthanized via cervical dislocation prior to tissue sample collection. There were no surviving animals at the end of study.

\section{Antibodies}

Rabbit anti-CCT6B (NBP2-92177) was purchased from Novus. Rabbit anti- $\beta$-TUBULIN was purchased from ABways (AB0039). LIN28A (ab46020) and anti- $\gamma \mathrm{H} 2 \mathrm{AX}$ (ab26350) were purchased from Abcam. Mouse anti-AC-Tubulin (T6793) was purchased from Sigma. Rabbit antiSOX9 (AB5535) was purchased from Merck.

\section{CRISPR/Cas9-mediated $\boldsymbol{C}_{\boldsymbol{C}} \mathbf{6} \boldsymbol{b}^{-/-}$mouse generation}

$C c t 6 b$-knockout mice were generated via a CRISPR/Cas9 approach as detailed previously (Wang et al. 2020; Zhang et al. 2019; Zhu et al. 2020). Briefly, single-guide RNAs (sgRNAs) targeting CCT6B exon 4 were designed with the following sequences: $5{ }^{\circ}$ GACGAAAGTTCATGCTGAACTGG-3' and 5'- ATGTTCTAGCCACATCCAAGAGG-3`. The Cas 9 and sgRNA plasmids were respectively linearized using AgeI and DraI respectively, and were then purified with a MinElute PCR Purification Kit (Qiagen, Duesseldorf, Germany). A MESSAGE mMACHINE T7 Ultra Kit (Ambion, TX, USA) was used to generate the Cas9 mRNA, while a MEGA Shortscript and Clear Kit (Ambion) was used to prepare the purified sgRNA. Wildtype C57BL/6 superovulated females were then mated with C57BL/6 males to generate zygotes for Cas9 mRNA and sgRNA injection.

\section{T7EI cleavage assay and sequencing}

Genomic DNA (gDNA) was extracted from testes using the Universal Genomic DNA kit (CW2298M; CWBIO) and amplified using Phanta Max super-fidelity DNA polymerase (p525; VAZYME) and the primers listed in Supplementary Table 2. After agarose gel electrophoresis, PCR products were purified using a PCR cleanup kit (AP-PCR-250; Axygen). A T7EI cleavage assay was then used for genotyping as described previously (Shen et al. 2013). Briefly, after having been mixed with Buffer 2 (NEB), purified PCR products were denatured and re-annealed using a thermocycler. Then, the PCR products were digested using T7EI (M0302L; NEB) for 25 min at $37^{\circ} \mathrm{C}$ and separated on a $2.3 \%$ agarose gel. 
120

121

122

123

124

125

126

127

128

129

130

131

132

133

134

135

136

137

138

139

140

141

142

143

144

145

146

147

148

149

150

151

152

153

154

155

156

157

158

159

\section{Off-target effect assay}

Using the open tool CRISPOR (http://crispor.tefor.net/) (Concordet \& Haeussler 2018), we predicted potential off-target sites based on the sequence of the target site. Off-target site selection was performed as described previously (Niu et al. 2014). Briefly, the aNy base-GuanosineGuanosine (NGG) sequence was chosen as the protospacer adjacent motif (PAM), and sites with eight conserved base pairs proximal to the PAM with three or fewer total mismatches were chosen as potential off-target sites. Potential off-target loci were first amplified from gDNA extracted from the testes of founder mice with the primers listed in Supplementary Table 2. PCR fragments including the off-target loci were then subjected to the T7EI cleavage assay. Sanger sequencing of PCR products with typical T7EI cleavage bands was then performed.

\section{Genotyping}

Edited founders harboring $C c t 6 b$ frameshift mutations were mated with wild type mice for a minimum of three generations to eliminate the potential effects of off-target gene editing. PCR amplification was used to confirm the genotypes of the resultant offspring (primers: forward, $5^{\circ}$ GCATACTTACTACTCGGAGAGCAT $-33^{`} \quad$ reverse, 5 CAGAGATAAGAAGGTGGCATTGGA -3'), and Sanger sequencing was additionally conducted, with the results being analyzed via SnapGene (v.3.2.1).

\section{qPCR}

An RNeasy Plus Micro Kit with on-column DNase digestion (Qiagen Ltd., 74034) was used to extract RNA from murine tissues. A heat-sterilized Teflon micropestle containing 350 $\mu$ l of RLT buffer and $4 \mu \mathrm{l}$ of $\beta$-mercaptoethanol was used to homogenize samples, after which RNA was isolated based on provided directions, with samples being maintained on ice at all times. Final RNA samples were eluted in $14 \mu \mathrm{l}$ of RNase-free water, after which $1 \mu \mathrm{g}$ of total RNA per sample was used to prepare cDNA with a PrimeScript RT reagent Kit (TaKaRa Bio Inc., RR037A). Both oligo(dT) and random primers were used for the reverse transcription. After a 15 min incubation at $37^{\circ} \mathrm{C}$, reverse transcriptase was heat-inactivated for $5 \mathrm{~s}$ at $85^{\circ} \mathrm{C}$. The samples were maintained on ice throughout the RNA extraction and reverse transcription. A qPCR instrument (StepOnePlus, Applied Biosciences) was then used to conduct triplicate gene expression analyses via a SYBR green approach. All qPCR primers are compiled in Supplementary Table 1, and 18S rRNA served as a normalization control.

\section{Western blotting}

Western blotting was conducted using a slightly modified version of a previously published protocol (Zheng et al. 2015). Briefly, a lysis buffer (7M urea, 2M thiourea, 2\% (w/v) DTT) containing a $1 \%(\mathrm{v} / \mathrm{w})$ protease inhibitor mixture (Pierce Biotechnology) was used to extract proteins, which were subsequently separated via SDS-PAGE and transferred to PVDF membranes. These blots were then blocked for $2 \mathrm{~h}$ at room temperature with 5\% nonfat milk in TBST, followed 
160 by overnight $(>12 \mathrm{~h})$ incubation with primary antibodies at $4^{\circ} \mathrm{C}$. Anti-CCT6B was used at a 1:1000 161 dilution, while anti- $\beta$-TUBULIN was used at a 1:3000 dilution. After three washes with TBST, 162 blots were then probed for $2 \mathrm{~h}$ with secondary antibodies, after which the SuperSignalWest Femto 163 Chemiluminescent Substrate Western Blotting detection system (Thermo Scientific) was used to 164 detect protein bands.

165

166

167

168

169

170

171

172

173

174

175

176

177

178

179

180

181

182

183

184

185

186

187

188

189

190

191

192

193

194

195

196

197

198

\section{Histological analyses}

Murine testes or epididymal tissues were collected from a minimum of three mice per genotype and were fixed for $24 \mathrm{~h}$ in modified Davidson's fluid prior to storage in $70 \%$ ethanol. A graded ethanol series was then used to dehydrate these samples, which were paraffin-embedded and cut to prepare $5 \mu \mathrm{m}$-thick sections that were mounted onto glass slides. Following deparaffinization, these sections were subjected to Periodic Acid Schiff or Hematoxylin and eosin (H\&E) staining to facilitate histological analyses.

\section{Sperm Analysis}

Sperm analyses were conducted as in prior reports (Castaneda et al. 2017; Jiang M 2014). Briefly, distal and clamping of the cauda region of the right epididymis was conducted for each mouse, after which this section was excised, washed with warm PBS, and added to an Eppendorf tube containing fresh human tubal fluid (HTF) media (Millipore) containing $10 \% \mathrm{FBS}$ at $37^{\circ} \mathrm{C}$. Clamping was then reversed, and the cauda was pierced with the tip of a scalpel to enable the sperm to diffuse into the medium for $5 \mathrm{~m}$ in at $37^{\circ} \mathrm{C}$. Sperm were then diluted using additional medium to enable a sperm motility analysis of a $10 \mu \mathrm{l}$ sperm suspension via computer-assisted semen analysis detection (Hamilton Thorne Research Inc.).

\section{Immunofluorescence staining}

Following deparaffinization, tissue sections were rehydrated, washed thrice with PBS (10 $\mathrm{min} /$ wash), and boiled in $10 \mathrm{mM}$ citrate buffer ( $\mathrm{pH} \mathrm{6.0)}$ in a microwave to facilitate antigen retrieval. A microwave oven for $10 \mathrm{~min}$. For spermatozoa samples, preparation was instead conducted by spreading cells onto microscope slides and allowed to air-dry. These cells were then fixed for 10 min using 1\% paraformaldehyde in PBS.

Immunofluorescent staining was conducted by washing samples thrice with PBST (10 $\mathrm{min} /$ wash), followed by a $1 \mathrm{~h}$ blocking step using $1 \%$ BSA. Samples were then incubated overnight $\left(>12 \mathrm{~h}\right.$ ) with appropriate primary antibodies at $4^{\circ} \mathrm{C}$, followed by incubation for $2 \mathrm{~h}$ with secondary antibodies. Anti-LIN28A was used at 1:500, anti-SOX9 at 1:500, anti- $\gamma \mathrm{H} 2 \mathrm{AX}$ was used at 1:100, and anti-AC-TUBULIN was used at 1:500. Hoechst 33342 was used to counterstain nuclei for 5 min, after which slides were rinsed with PBS and mounted using VectaShield or Immu-Mount. An LSM800 confocal microscope (Carl Zeiss AG) was then used to image the stained slides.

\section{Transmission electron microscopy}


199

200

201

202

203

204

205

206

207

208

209

210

211

212

213

214

215

216

217

218

219

220

221

222

223

224

225

226

227

228

229

230

231

232

233

234

235

236

237

238

Ultrastructural analyses were performed as in prior reports (Hua et al. 2019). Briefly, testes from adult mice were isolated and fixed overnight using $2.5 \%(\mathrm{v} / \mathrm{v})$ glutaraldehyde in $0.2 \mathrm{M}$ cacodylate buffer (50 mM cacodylate, $50 \mathrm{mM} \mathrm{KCl}$, and $2.5 \mathrm{mM} \mathrm{MgCl} 2, \mathrm{pH}$ 7.2). After subsequent washing in this buffer, the tissues were cut into $\sim 1 \mathrm{~mm}^{3}$ pieces and submerged in $1 \%$ OsO4 in 0.2 $\mathrm{M}$ cacodylate buffer for $2 \mathrm{~h}$ at $4^{\circ} \mathrm{C}$. Samples were then washed again prior to overnight immersion in $0.5 \%$ uranyl acetate. After dehydration with an ethanol gradient, these samples were embedded in resin using the Low Viscosity Embedding Media Spurr's Kit (EMS, 14300). An ultramicrotome was then used to prepare ultrathin sample sections that were mounted onto copper grids, stained for 10 min with lead citrate and uranyl acetate, and assessed with a JEM-1400 transmission electron microscope (JEOL).

\section{Statistical analysis}

Data are means $\pm \mathrm{SEM}$, and were compared via two-tailed Student's t-tests. Not significant (NS): $\mathrm{P} \geq 0.05 ; * \mathrm{P}<0.05 ; * * \mathrm{P}<0.01 ; * * * \mathrm{P}<0.001 ; * * * * \mathrm{P}<0.0001$.

\section{Results}

\section{Assessment of murine CCT6B expression patterns}

Given that Cct6b is evolutionarily conserved (Supplementary Fig. S1), it is likely to play conserved functions in animals expressing this gene. To explore these functions, we assessed $C c t 6 b$ expression patterns in mice via qPCR, revealing abundant expression of this gene in the testis but not in other analyzed tissues (Fig. 1A). The first round of spermatogenesis in murine testis is relatively synchronous. As such, we collected testis tissue Cct6b expression in mice at different numbers of weeks after birth (W) in order to capture the initial spermatogenesis wave. This analysis revealed Cct6b expression beginning at week 3, which is the developmental stage during which spermatids emerge in murine testes (Fig. 1B).

\section{Cct6b $^{-/}$mouse generation}

To explore the functional importance of $C c t 6 b$ in spermatogenesis, we next used a CRISPR/Cas9 approach to generate C57BL/6 mice in which this gene had been knocked out. To accomplish this, a frameshift mutation in exon 4 of the $C c t 6 b$ gene was introduced into superovulated fertilized mouse eggs (Fig. 2A). The mutation causes p.Glu125Gly fs ter22 (Supplementary Fig. S2), and has the potential to result in the nonsense-mediated decay of the encoded mRNA. Relative to wild-type controls, animals in the F2 $C c t 6 b^{-/}$generation harbored a 5 bp deletion in exon 4 of $C c t 6 b$ as detected by PCR and Sanger sequencing (Fig. 2B). Western blotting and qPCR further confirmed that the expression of Cct6b was completely absent at the protein level and markedly reduced at the mRNA level in the testes of $C c t 6 b^{-/}$mice (Fig. 2C-D). Concerns involving off-target mutations associated with the CRISPR/Cas9 system have been previously expressed (Pattanayak et al. 2013; Sander \& Joung 2014), and as such, we analyzed these animals for any potential off-target events. We first excluded the potential off-target effects 
275

276

277

278

of the sgRNAs on other CCT genes. Although there is a high degree of homology among the CCT family proteins, there were still multiple mismatched base pairs at the sgRNA site (Figure. S3A). More importantly, protospacer adjacent motif (PAM) sequences at the sgRNA sites of other CCT family members are not conserved, and these play a crucial role in the efficiency of sgRNA cleavage (Figure. S3A) (Cong et al. 2013; Mojica et al. 2009). In addition, predicted off-target sites within the genome were identified, and a subsequent T7E1 assay exhibited no off-target effects on these sites (Supplementary Table3 and Fig. S3B). The Cct $6 b^{-/}$mice were viable and developmentally normal. Collectively, these results indicated that we had successfully constructed a Cct6b-knockout mouse line.

\section{$\operatorname{Cct}^{-/ /}$mice exhibit normal spermatogenesis}

The fertility of male mice was next assessed by housing $C c t 6 b^{+/+}$or $C c t 6 b^{-/-}$males with wildtype females for 4 months and recording the number of offspring per litter. The average number of pups per litter for $C c t 6 b^{+/+}$mating pairs was $4.5 \pm 1.22$, while $C c t 6 b^{-/}$males sired an average of $5 \pm 1.87$ pups per litter. This suggests that $C c t 6 b$ is dispensable for male fertility (Fig. 3A). No differences in testes $(\mathrm{n}=3)$ or epididymis (Fig. 3B-C) size were observed when comparing mice in these two groups, and PAS staining did not reveal any differences in spermatogenesis when assessing testes or epididymal tissues from these mice. The average numbers of spermatocytes, round spermatids, and elongated spermatids per tubule were comparable when comparing adult wild-type and $\mathrm{Cct}_{6} \mathrm{~b}^{-/}$testes (Fig. 3D-E). In addition, immunofluorescent staining revealed normal spermatogonia self-renewal, meiotic progression, and acrosome formation in these two groups (Fig. 4A-C), and comparable SOX9 expression was evident in $\mathrm{Cct}_{6 b^{+/+}}$and $\mathrm{Cct}_{6} 6 b^{-/}$mice (Fig. 4D-E). Together, these results indicated that $C c t 6 b^{-/}$mice are fertile and exhibit normal spermatogenesis.

\section{CCT6B knockout results in the bending of the sperm neck}

After developing in the testes, spermatozoa enter the epididymis. However, no differences were observed when assessing H\&E-stained epididymal samples from these two groups of mice (Fig. 5A). We therefore directly assessed sperm quality in $C c t 6 b^{-/}$males with a Computer-Assisted Sperm Analyzer (CASA). The overall epididymal cauda sperm counts were comparable for $\mathrm{Cct} 6 \mathrm{~b}^{+/+}$and $\mathrm{Cct} 6 \mathrm{~b}^{-/}$animals (Fig. 5B), and sperm motility was also similar in these two groups (Fig. 5C). However, sperm from $\mathrm{Cct}_{6} b^{-/}$mice exhibited significantly decreased progressive motility (Fig. 5D). Morphological analyses also revealed that the ratio of normal sperm ( $\mathrm{n}=3, P=$ 0.006) was significantly decreased in male $C c t 6 b^{-/}$mice, with nuclear base bending being the primary abnormality evident in these mice $(\mathrm{n}=3, P=0.005)$ (Fig. 5E-F). However, immunofluorescent staining suggested that the major structures of these malformed sperm, including the flagella and acrosome, remained normal (Fig. 5G). To assess whether the occurrence of bent neck $C c t 6 b^{-/}$sperm was the result of defective flagellar organization, we employed TEM to directly evaluate sperm morphology. No significant differences were observed when comparing flagella between $C c t 6 b^{-/}$and $C c t 6 b^{+/+}$sperm, but cytoplasmic retention around the head of $C c t 6 b^{-/-}$ 
279

280

281

282

283

284

285

286

287

288

289

290

291

292

293

294

295

296

297

298

299

300

301

302

303

304

305

306

307

308

309

310

311

312

313

314

315

316

317

318

sperm was observed via this approach, as was the curling of the flagellar tail in the cytoplasm (Fig. 6). Together, these results indicated that $C c t 6 b$ knockout can result in teratospermia characterized by neck bending and cytoplasmic redundancy.

\section{Other CCT complex components exhibit decreased transcript abundance in $\boldsymbol{C c t}_{\boldsymbol{~}} \boldsymbol{b}^{-/-}$mice}

Given the function of CCT6B as a CCT complex protein, and the high degree of homology between CCT6B and the other CCT proteins, we additionally explored the impact of knocking out this gene on other CCT complex components at the mRNA level. Compared with the control group, the mRNA expression levels of other CCT family genes were not significantly increased in Cct6 $^{-/}$testes, (Fig. 7), suggesting that the loss of function of CCT6B was not compensated for by the upregulation of other CCT complex components.

\section{Discussion}

The CCT chaperone complex is estimated to aid in the folding of up to $15 \%$ of the eukaryotic proteome (Thulasiraman et al. 1999; Yam et al. 2008). Over half of active chaperons are occupied by the folding of tubulin and actin, which serve as the primary components of microtubules and the acrosome, respectively, suggesting a potential mechanistic relationship between CCT and spermiogenesis (Siegers et al. 2008). Indeed, prior reports have shown CCT proteins to play important roles in spermatogenesis and fertilization. Sylvie et al. initially demonstrated the presence of CCT in the microtubule-organizing centers (MTOCs) and manchette microtubules during spermiogenesis, indicating that this chaperone complex is likely a key regulator of sperm microtubule assembly (Souès et al. 2003). Matthew et al. determined that CCT is present on capacitated spermatozoa surfaces and may regulate the zona binding activity of these cells (Dun et al. 2011). CCT knockdown experiments have recently revealed that knocking down any CCT complex subunits can adversely affect fertility in planarians (Counts et al. 2017). However, no knockout studies in mammalian models have specifically explored the role of CCT in spermatogenesis.

CCT6B is a member of the CCT complex that exhibits a testis-specific expression pattern. Herein, we confirmed that this gene was expressed exclusively during spermiogenesis (Fig. 1), indicating that CCT6B is likely to play an important role in regulating spermiogenesis, spermatid release, and maturation. To test the functional importance of this gene, we generated $C c t 6 b$ knockout mice via a CRISPR/Cas9 approach and confirmed that CCT6B protein expression was absent in the testes of these animals (Fig. 2). Adult male $C_{c t} 6 b^{-/}$C57BL/6 mice remained fertile, and no differences in the histology or morphology of the testes and epididymides of these mice were observed when compared to wild-type controls (Fig. 3). Spermatogonia proliferation and spermatocyte meiosis also appeared normal in these $C c t 6 b^{-/}$animals (Fig. 4), and epididymal sperm numbers and motility remained normal. However, progressive motility and the ratio of normal sperm were significantly decreased in male $C c t 6 b^{-/}$mice (Fig. 5). TEM revealed a deficiency in the neck of $C c t 6 b^{-/}$sperm (Fig. 6). As CCT paralogs share $65-71 \%$ sequence identity across most of their exonic sequences, with the ubiquitously-expressed Cct6a protein 
exhibiting particularly high levels of homology with Cct6b (Counts et al. 2017), we additionally evaluated the expression of the other CCT proteins. This analysis revealed slight reductions in Cct4 and Cct7 levels, whereas no changes in other components were detected (Fig. 7), suggesting that no compensatory effects mediated by the expression of other CCT complex components occur in these tissues.

Our results indicate that $\mathrm{CCT} 6 \mathrm{~B}$ plays an important role in regulating sperm morphogenesis. The loss of CCT6B results in an increased ratio of sperm nuclear base bending, suggesting that the functionality of certain structural proteins in these cells is disrupted. This highlights a potential role for the CCT complex in the assembly of structural proteins during spermatogenesis. Even so, CCT6B-deficient mice generated normal amounts of sperm and remained fertile, suggesting that CCT6B is an auxiliary module in the CCT complex, consistent with its testis-specific expression pattern. One possibility is that it plays an enhanced role in protein folding processes in the context of spermatogenesis, but further research will be required to test this possibility.

\section{Acknowledgements}

We thank Jinyang Cai for continuous support with microscopy.

\section{References}

Broadley S, and Hartl F. 2009. The role of molecular chaperones in human misfolding diseases. FEBS letters 583:2647-2653.

Castaneda J, Hua R, Miyata H, Oji A, Guo Y, Cheng Y, Zhou T, Guo X, Cui Y, Shen B et al. . 2017. TCTE1 is a conserved component of the dynein regulatory complex and is required for motility and metabolism in mouse spermatozoa. Proc Natl Acad Sci U S A 114:E5370-E5378.

Concordet J, and Haeussler M. 2018. CRISPOR: intuitive guide selection for CRISPR/Cas9 genome editing experiments and screens. Nucleic acids research 46:W242-W245.

Cong L, Ran F, Cox D, Lin S, Barretto R, Habib N, Hsu P, Wu X, Jiang W, Marraffini L et al. . 2013. Multiplex genome engineering using CRISPR/Cas systems. Science (New York, NY) 339:819-823.

Counts J, Hester T, and Rouhana L. 2017. Genetic expansion of chaperonin-containing TCP-1 (CCT/TRiC) complex subunits yields testis-specific isoforms required for spermatogenesis in planarian flatworms. Molecular reproduction and development 84:1271-1284.

de Kretser D, Loveland K, Meinhardt A, Simorangkir D, and Wreford N. 1998. Spermatogenesis. Human reproduction (Oxford, England):1-8.

Dias G, Lino-Neto J, Mercati D, and Dallai R. 2016. The sperm structure and spermiogenesis of the heteropteran Coptosoma scutellatum (Geoffroy) with emphasis on the development of the centriole adjunct. Micron 82:33-40.

Dun M, Smith N, Baker M, Lin M, Aitken R, and Nixon B. 2011. The chaperonin containing TCP1 complex (CCT/TRiC) is involved in mediating sperm-oocyte interaction. J Biol Chem 286:36875-36887.

Dunn A, Melville M, and Frydman J. 2001. Review: cellular substrates of the eukaryotic chaperonin TRiC/CCT. Journal of structural biology 135:176-184. 
364

365

366

367

368

369

370

371

372

373

374

375

376

377

378

379

380

381

382

383

384

385

386

387

388

389

390

391

392

393

394

395

396

397

398

399

400

401

402

403

404

405

406

407

408

409

410

411

412

413

414

Fouquet J, and Kann M. 1994. The cytoskeleton of mammalian spermatozoa. Biology of the cell 81:89-93.

Giuffrida V, Pezzino F, Romano F, Litrico L, Garofalo M, Nicotra G, Libra M, D'Amico F, Castrogiovanni P, Imbesi R et al. . 2006. Gene expression in mouse spermatogenesis during ontogenesis. International journal of molecular medicine 17:523-528.

Hess R, and Renato de Franca L. 2008. Spermatogenesis and cycle of the seminiferous epithelium. Advances in experimental medicine and biology 636:1-15.

Hua R, Wei H, Liu C, Zhang Y, Liu S, Guo Y, Cui Y, Zhang X, Guo X, Li W et al. . 2019. FBXO47 regulates telomere-inner nuclear envelope integration by stabilizing TRF2 during meiosis. Nucleic acids research 47:11755-11770.

Jiang M GM, Wu C, He H, Guo X, Zhou Z, Yang H, Xiao X, Liu G, Sha J. 2014. Lack of testicular seipin causes teratozoospermia syndrome in men. Proc Natl Acad Sci U S A:7054-7059.

Kubota H, Hynes G, Carne A, Ashworth A, and Willison K. 1994. Identification of six Tcp-1related genes encoding divergent subunits of the TCP-1-containing chaperonin. Current biology : CB 4:89-99.

Kubota H, Hynes G, Kerr S, and Willison K. 1997. Tissue-specific subunit of the mouse cytosolic chaperonin-containing TCP-1. FEBS letters 402:53-56.

Kubota H, Hynes G, and Willison K. 1995a. The chaperonin containing t-complex polypeptide 1 (TCP-1). Multisubunit machinery assisting in protein folding and assembly in the eukaryotic cytosol. European journal of biochemistry 230:3-16.

Kubota H, Hynes G, and Willison K. 1995b. The eighth Cct gene, Cctq, encoding the theta subunit of the cytosolic chaperonin containing TCP-1. Gene 154:231-236.

Lopez T, Dalton K, and Frydman J. 2015. The Mechanism and Function of Group II Chaperonins. Journal of molecular biology 427:2919-2930.

Mali P, Esvelt K, and Church G. 2013. Cas9 as a versatile tool for engineering biology. Nature methods 10:957-963.

Mojica F, Díez-Villaseñor C, García-Martínez J, and Almendros C. 2009. Short motif sequences determine the targets of the prokaryotic CRISPR defence system. Microbiology (Reading, England) 155:733-740.

Niu Y, Shen B, Cui Y, Chen Y, Wang J, Wang L, Kang Y, Zhao X, Si W, Li W et al. . 2014. Generation of gene-modified cynomolgus monkey via Cas9/RNA-mediated gene targeting in one-cell embryos. Cell 156:836-843.

Pattanayak V, Lin S, Guilinger J, Ma E, Doudna J, and Liu D. 2013. High-throughput profiling of off-target DNA cleavage reveals RNA-programmed Cas9 nuclease specificity. Nature biotechnology 31:839-843.

Rothman J. 1989. Polypeptide chain binding proteins: catalysts of protein folding and related processes in cells. Cell 59:591-601.

Sander J, and Joung J. 2014. CRISPR-Cas systems for editing, regulating and targeting genomes. Nature biotechnology 32:347-355.

Shen B, Zhang J, Wu H, Wang J, Ma K, Li Z, Zhang X, Zhang P, and Huang X. 2013. Generation of gene-modified mice via Cas9/RNA-mediated gene targeting. Cell Res 23:720-723.

Siegers K, Bölter B, Schwarz J, Böttcher U, Guha S, and Hartl F. 2008. TRiC/CCT cooperates with different upstream chaperones in the folding of distinct protein classes. The EMBO journal 27:301.

Souès S, Kann M, Fouquet J, and Melki R. 2003. The cytosolic chaperonin CCT associates to cytoplasmic microtubular structures during mammalian spermiogenesis and to heterochromatin in germline and somatic cells. Exp Cell Res 288:363-373.

Spiess C, Meyer A, Reissmann S, and Frydman J. 2004. Mechanism of the eukaryotic chaperonin: protein folding in the chamber of secrets. Trends Cell Biol 14:598-604.

Peer) reviewing PDF | (2021:02:58098:1:0:NEW 22 Apr 2021) 
415 Thulasiraman V, Yang C, and Frydman J. 1999. In vivo newly translated polypeptides are sequestered in a protected folding environment. The EMBO journal 18:85-95.

Valpuesta J, Martín-Benito J, Gómez-Puertas P, Carrascosa J, and Willison K. 2002. Structure and function of a protein folding machine: the eukaryotic cytosolic chaperonin CCT. FEBS letters 529:11-16.

Wang H, Yang H, Shivalila C, Dawlaty M, Cheng A, Zhang F, and Jaenisch R. 2013. One-step generation of mice carrying mutations in multiple genes by CRISPR/Cas-mediated genome engineering. Cell 153:910-918.

Wang X, Xie W, Yao Y, Zhu Y, Zhou J, Cui Y, Guo X, Yuan Y, Zhou Z, and Liu M. 2020. Hspa1IThe heat shock protein family gene in male mice is dispensable for fertility. PeerJ 8:e8702.

Yam A, Xia Y, Lin H, Burlingame A, Gerstein M, and Frydman J. 2008. Defining the TRiC/CCT interactome links chaperonin function to stabilization of newly made proteins with complex topologies. Nat Struct Mol Biol 15:1255-1262.

Zhang J, Zhang X, Zhang Y, Zeng W, Zhao S, and Liu M. 2019. Fank1Normal spermatogenesis in (fibronectin type 3 and ankyrin repeat domains 1) mutant mice. PeerJ 7:e6827.

Zheng B, Zhao D, Zhang P, Shen C, Guo Y, Zhou T, Guo X, Zhou Z, and Sha J. 2015. Quantitative Proteomics Reveals the Essential Roles of Stromal Interaction Molecule 1 (STIM1) in the Testicular Cord Formation in Mouse Testis. Molecular \& cellular proteomics : MCP 14:2682-2691.

Zhu Y, Cui Y, Guo X, Wang L, Bi Y, Hu Y, Zhao X, Liu Q, Huo R, Lin M et al. . 2006. Proteomic analysis of effect of hyperthermia on spermatogenesis in adult male mice. Journal of proteome research 5:2217-2225.

Zhu Z, Zhang X, Zeng W, Zhao S, Zhou J, Zhou Z, and Liu M. 2020. Tex33Spermatogenesis is normal in knockout mice. PeerJ 8:e9629. 
Figure 1

Cct6b distribution patterns in mice.

(A) The expression of Cct6b in different murine tissues (12 weeks) was assessed via qPCR.

$n=3$. (B) The expression of $C c t 6 b$ in murine testes at different developmental stages, including $0,1,2,3,4,5,6$, and 7 weeks, was assessed via qPCR. 18s served as a normalization control for all qPCR analyses.
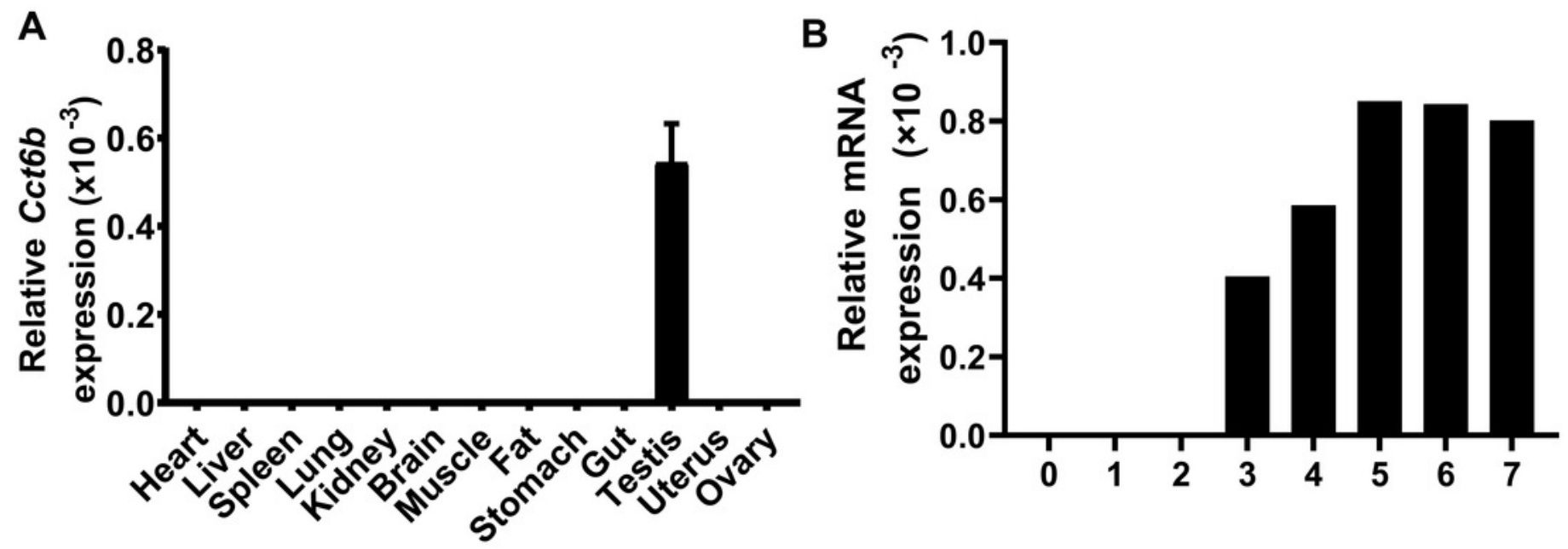
Figure 2

$\mathrm{Cct}^{-/} \mathrm{b}^{-/}$mouse generation.

(A) An overview of the CRISPR/Cas9 knockout targeting strategy. (B) Sanger sequencing indicated the presence of a 5-bp deletion in $C c t 6 b^{-/}$mice. (C) Cct6b expression was confirmed to be reduced via qPCR in $C c t 6 b^{-/}$mice. (D) Western blotting confirmed that testis samples from $\mathrm{Cct}_{6 b^{-/}}$mice did not exhibit a band of the expected size (58.1 kD). $\beta$-TUBULIN served as a normalization control.

A

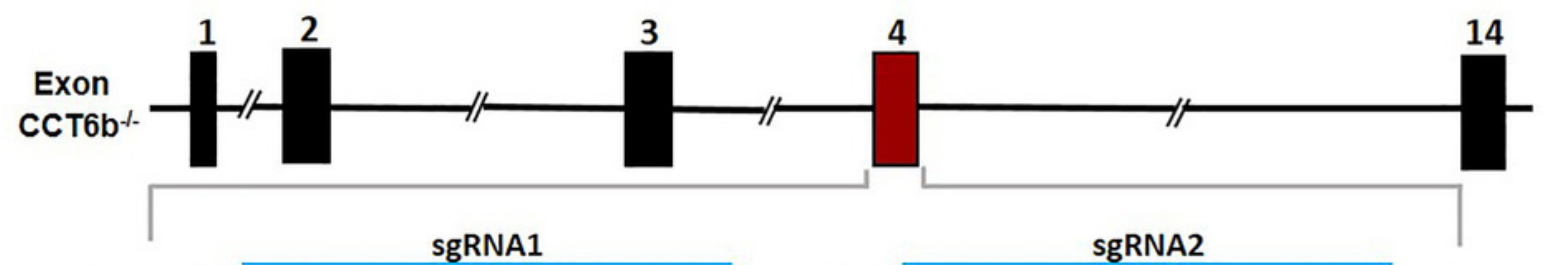

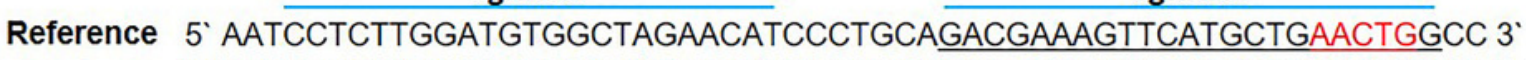

3`TTAGGAGAACCTACACCGATCTTGTAGGGACGTCTGCTTTCAAGTACGACTTGACCGG 5

c. $373 \_377$ del AACTG Glu 125 Gly fs Ter22

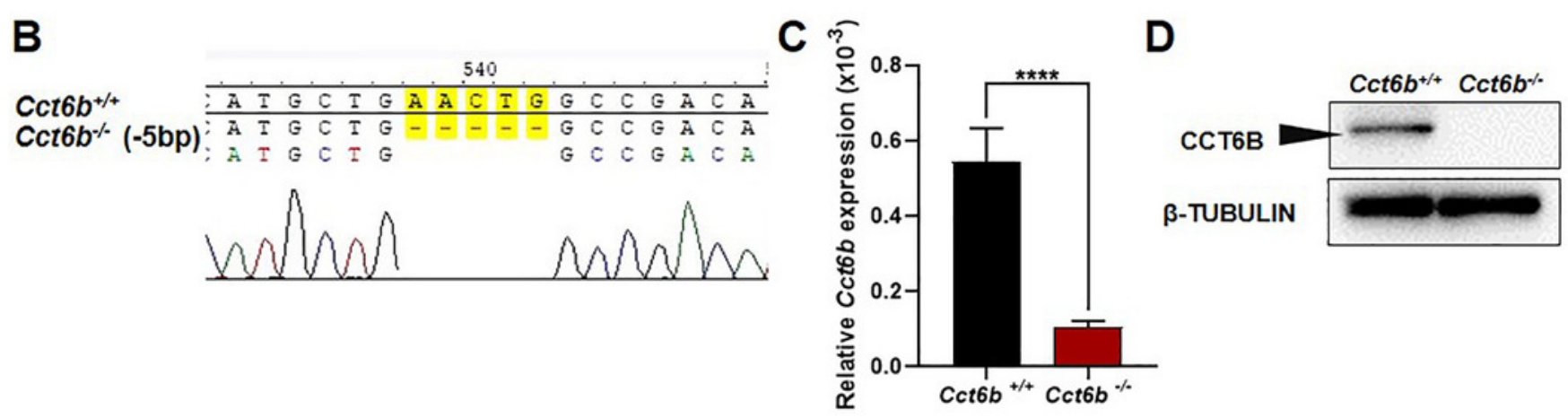




\section{Figure 3}

$C c t 6 b^{-/}$mice exhibit normal spermatogenesis.

(A) The average number of pups per litter for $\mathrm{Cct6}^{+/+}$and $C \operatorname{ct} 6 b^{-/-}$mice, $\mathrm{n}=3$. (B) Images of testis and epididymis samples from $C c t 6 b^{+/+}$and $C c t 6 b^{-/}$adult mice. (C) Average testis weight normalized to body weight, $\mathrm{n}=3$. (D) Testis sections from $C c t 6^{+/+}$and $C c t 6 b^{-/}$mice following Periodic-acid Schiff staining. (E) The relative composition ratios of different cell types in testis sections at spermatogenic stages VIII and XI. P-L, pre-leptotene; Z, zygotene; P, pachytene; D, diplotene; RS, round spermatids; ES, elongated spermatids. 

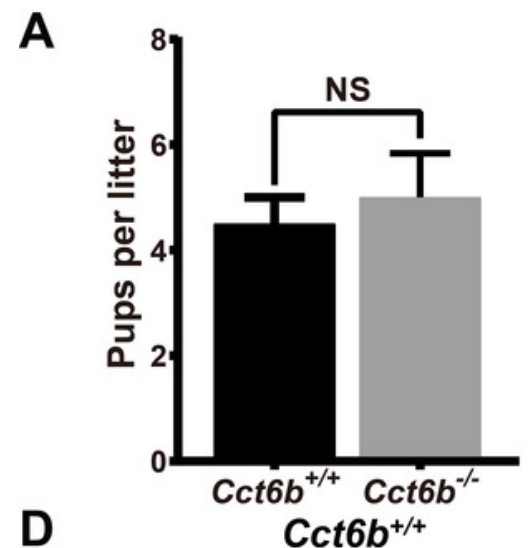

B

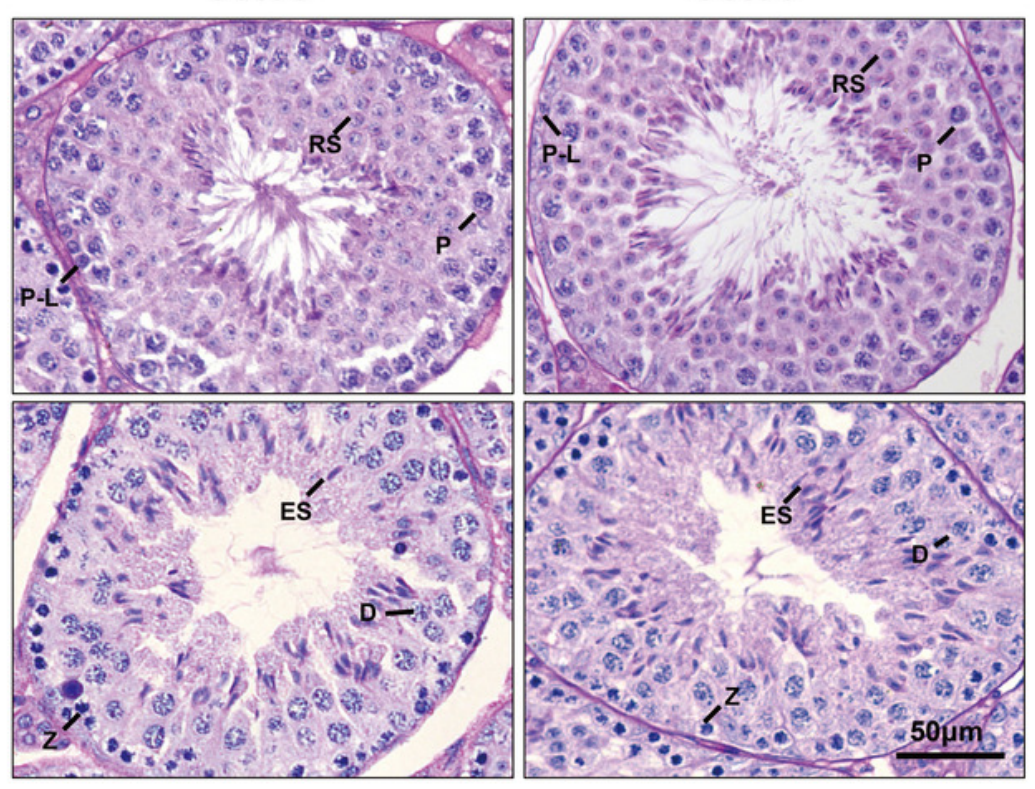

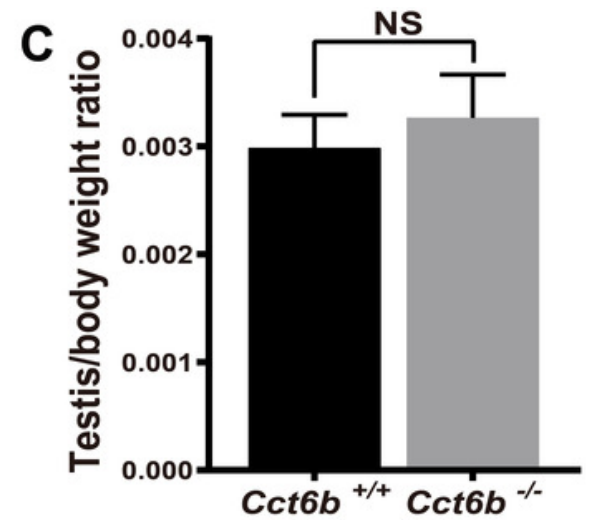
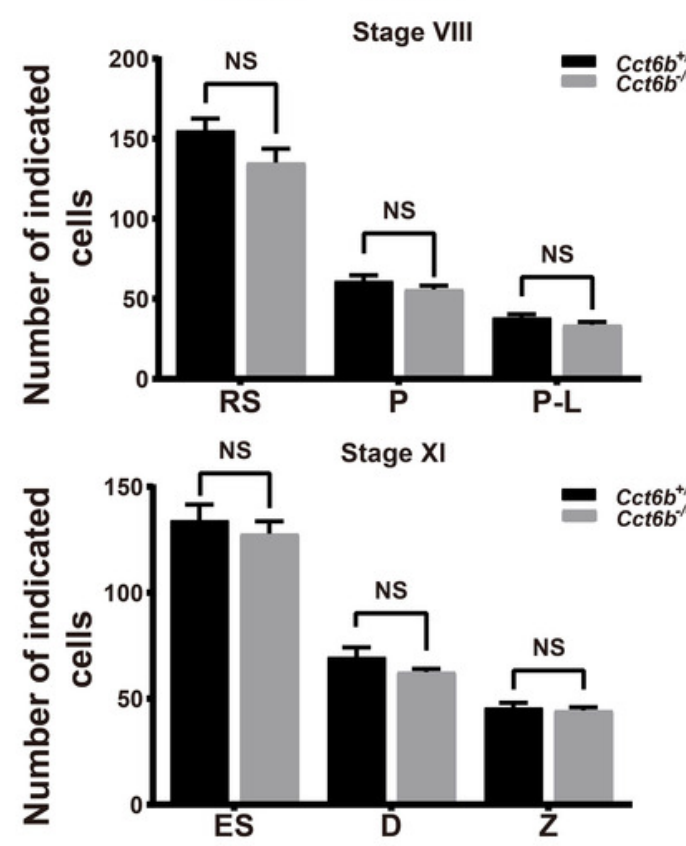


\section{Figure 4}

$C c t 6 b^{\%}$ mice exhibit normal spermatogenic marker expression.

(A) DNA double-strand break formation during meiosis, as measured via $\mathrm{YH} 2 \mathrm{AX}$ staining, appeared normal. Spermatids from these knockout mice also exhibited normal acrosomal structures (PNA); Z, zygotene; $P$, pachytene; $D$, diplotene; $n=3$. (B)-(C) Spermatogonia proliferation (LIN28A) did not differ significantly when comparing $\mathrm{CCT}_{6} \mathrm{~b}^{-/}$and $\mathrm{CCT}_{6} \mathrm{~b}^{+/+}$mice; Spg, spermatogonia, $n=3$. (D)-(E) Sertoli cells (SOX9) numbers in testis sections were comparable in $\mathrm{Cct6}^{+/+}$and $c c t 6 b^{-/}$mice. Ser, Sertoli cell, $\mathrm{n}=3$. 
A

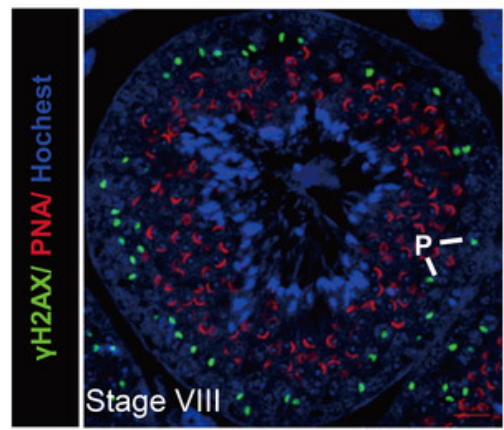

B

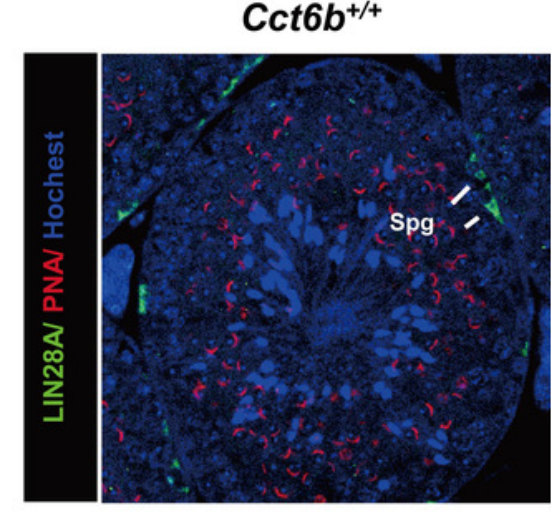

D

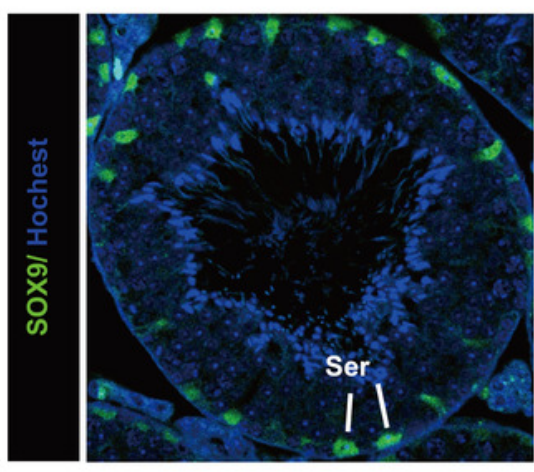

Cct6 $6 b^{+/+}$

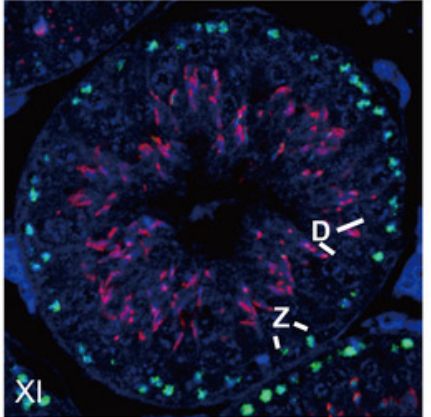

Cct6b $^{-/}$

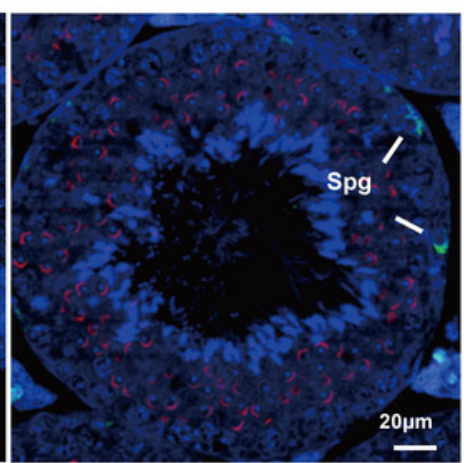

$\underline{20 \mu m}$

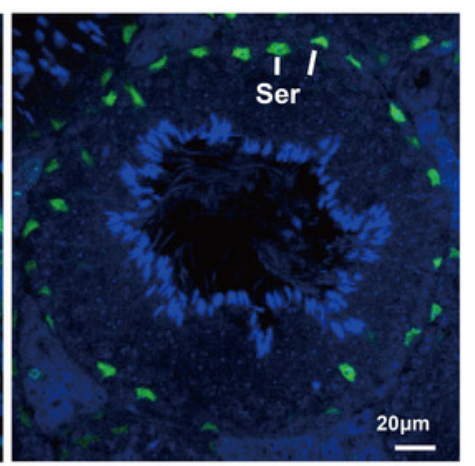

Cct6b--

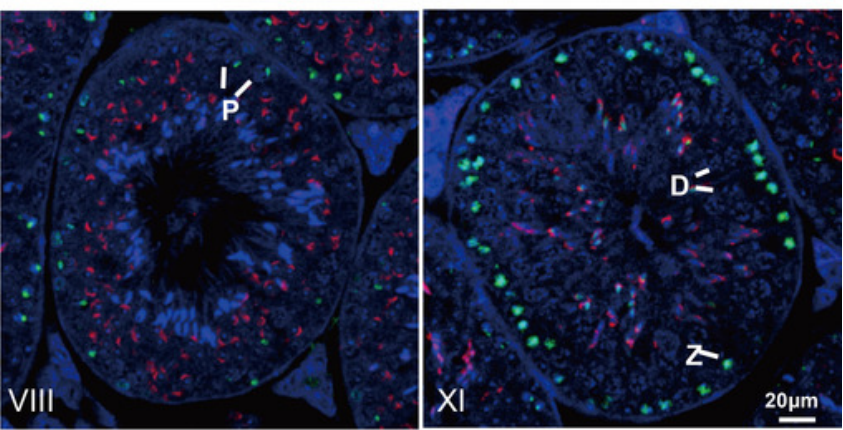

C

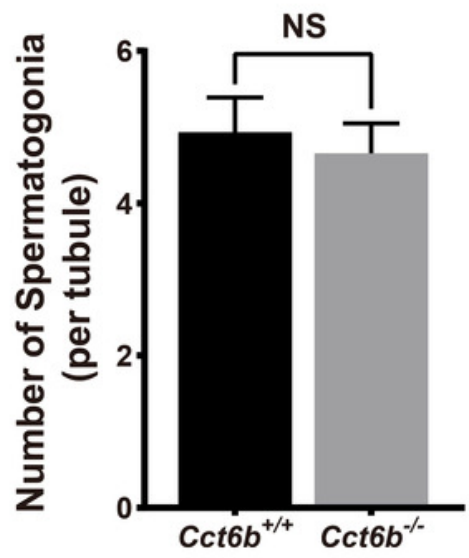

E

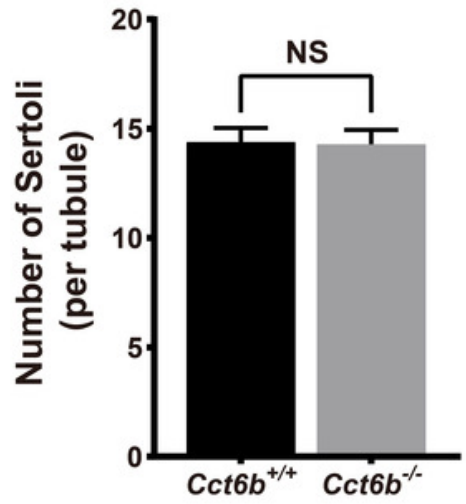




\section{Figure 5}

Teratozoospermia is evident in $C c t 6 b^{-/}$males.

(A) H\&E-stained cauda epididymis tissues from $C c t 6 b^{+/+}$and $C c t 6 b^{-/}$mice. (B) Cauda epididymal sperm contents from $C c t 6^{+/+}$and $C c t 6 b^{-/}$mice, $n=3$. (C) Average frequencies of motile and (D) progressive sperm in the $C c t 6^{+/+}$and $C c t 6 b^{-/}$mice, $n=3$. (E) H\&E-stained spermatozoa from $C c t 6^{+/+}$and $C c t 6 b^{-/}$mice. (F) Frequencies of sperm exhibiting abnormal morphological characteristics in $C c t 6^{+/+}$and $C c t 6 b^{-/}$mice, $n=3$. (G) AC-tubulin and PNA immunofluorescent detection in $\mathrm{Cct}^{+/+}$and $\mathrm{Cct} \mathrm{b}^{-/}$spermatozoa. 
A
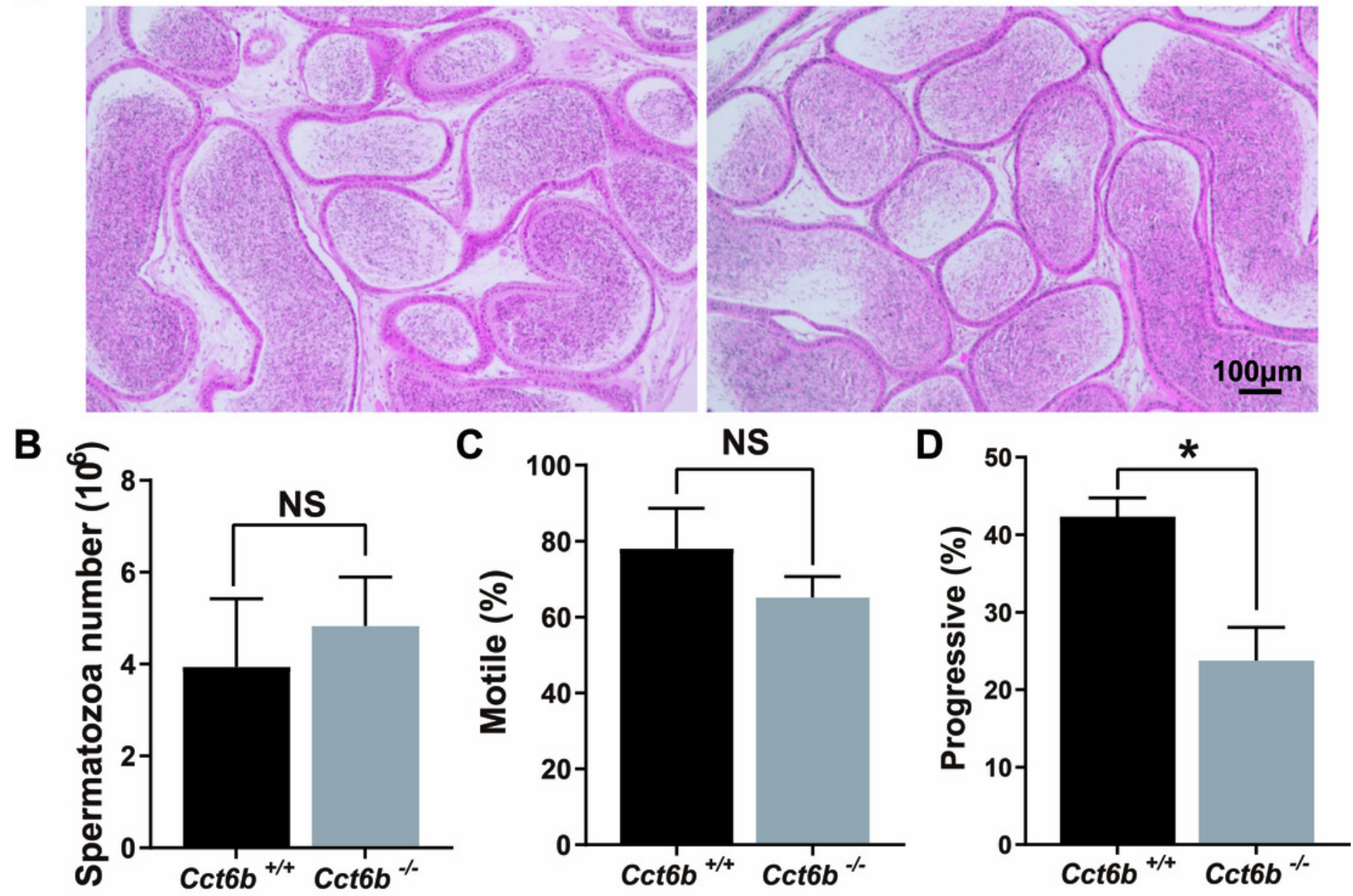

E

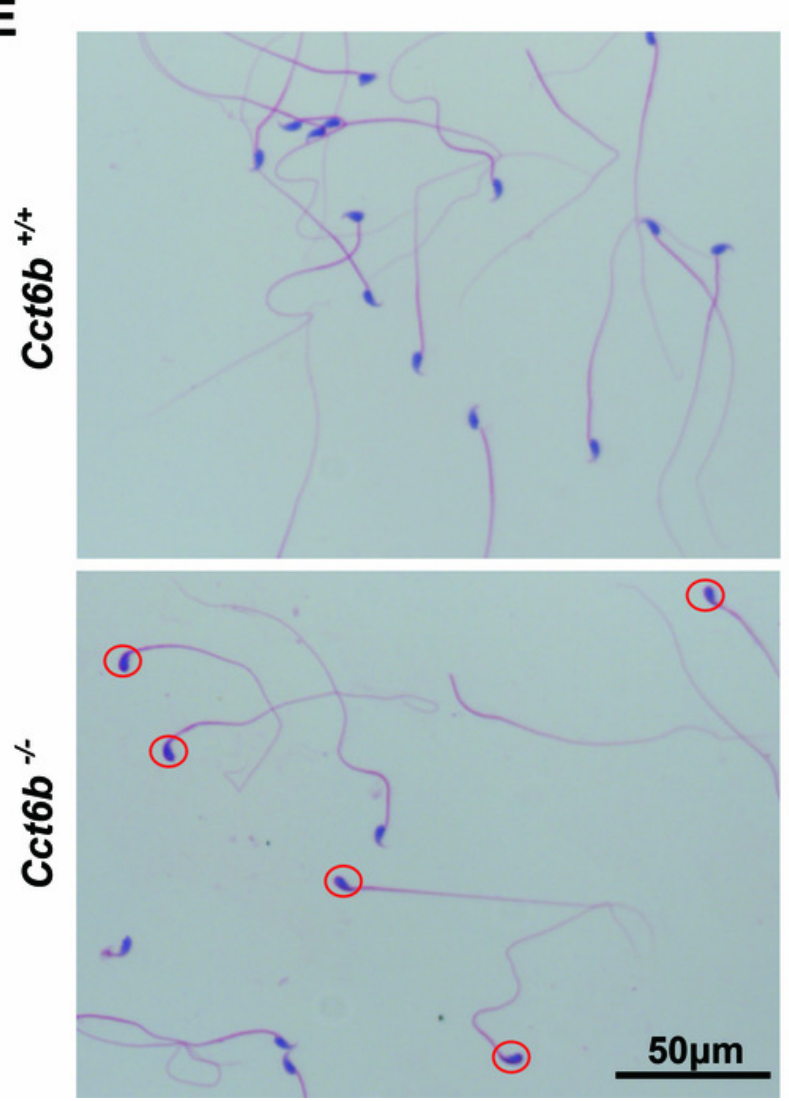

$\mathbf{F}$

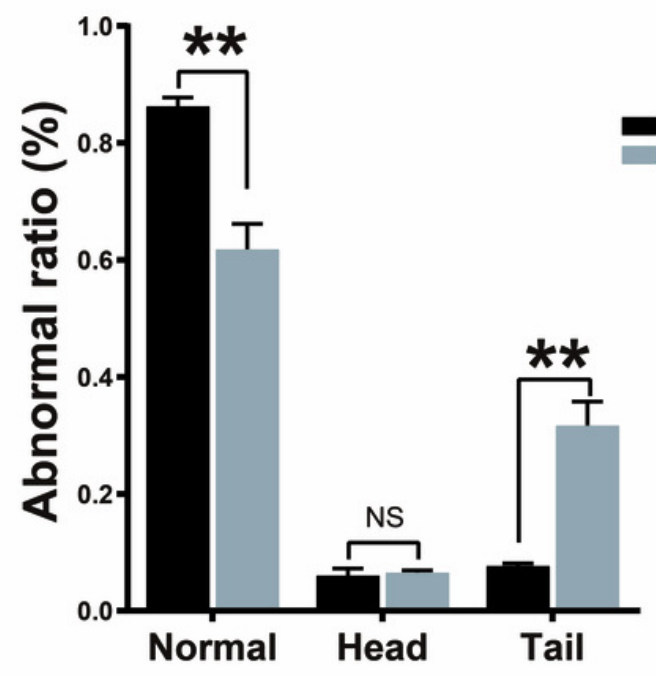

G

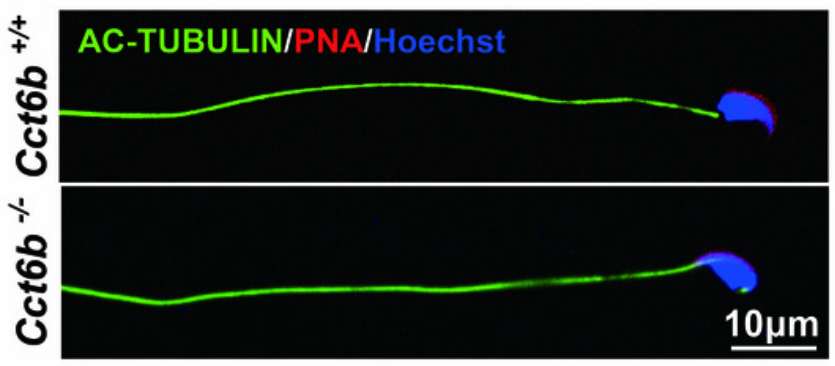




\section{Figure 6}

Electron microscopy images of $\mathrm{Cct6}^{+/+}$and $C c t 6 b^{-/}$spermatozoa.

The cytoplasm of knockout sperms remain around the head of the sperm, while the flagella remain curled in the cytoplasm of these cells (arrows); *, Residual cytoplasm; M, midpiece; P, principal piece.

$\mathrm{Cct} 6 \mathrm{~b}^{+/+}$

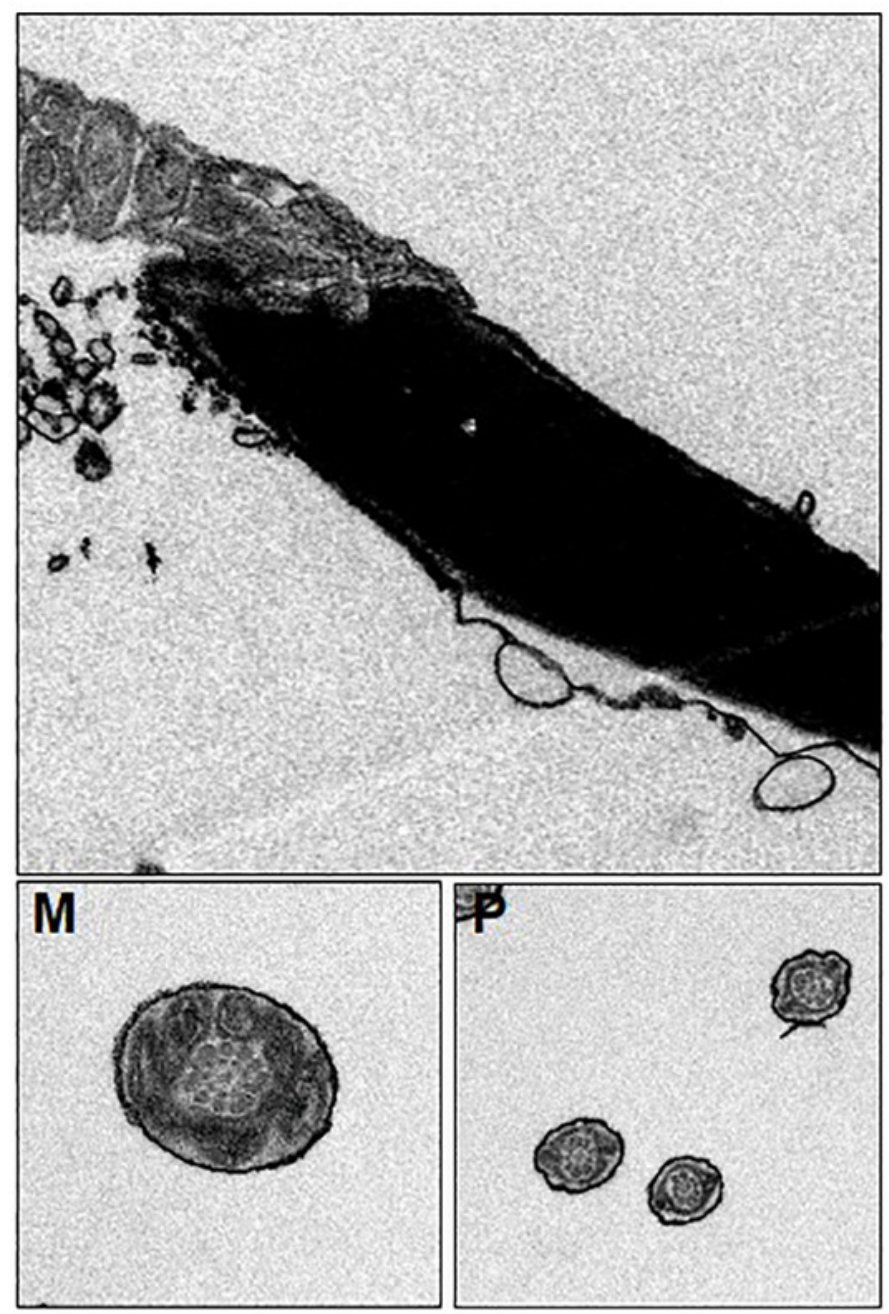

$\operatorname{Cct6}^{-/-}$
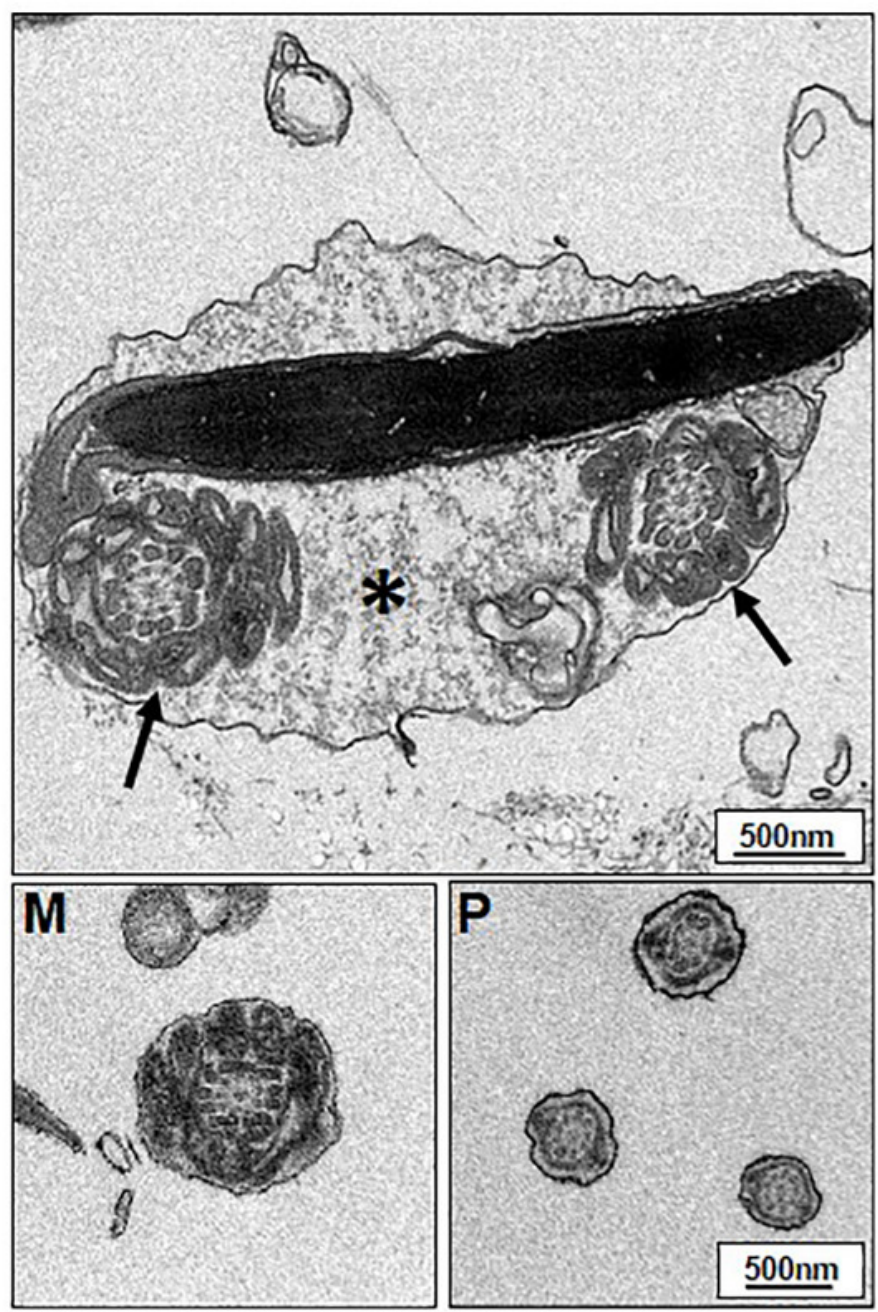
Figure 7

Gene expression changes in the testes of $c c t 6 b^{-/}$mice.

The expression of Cct1-5, Cct6a, and Cct7-8 in murine testes was assessed via qPCR. 18S rRNA served as a normalization control. $n=3$.

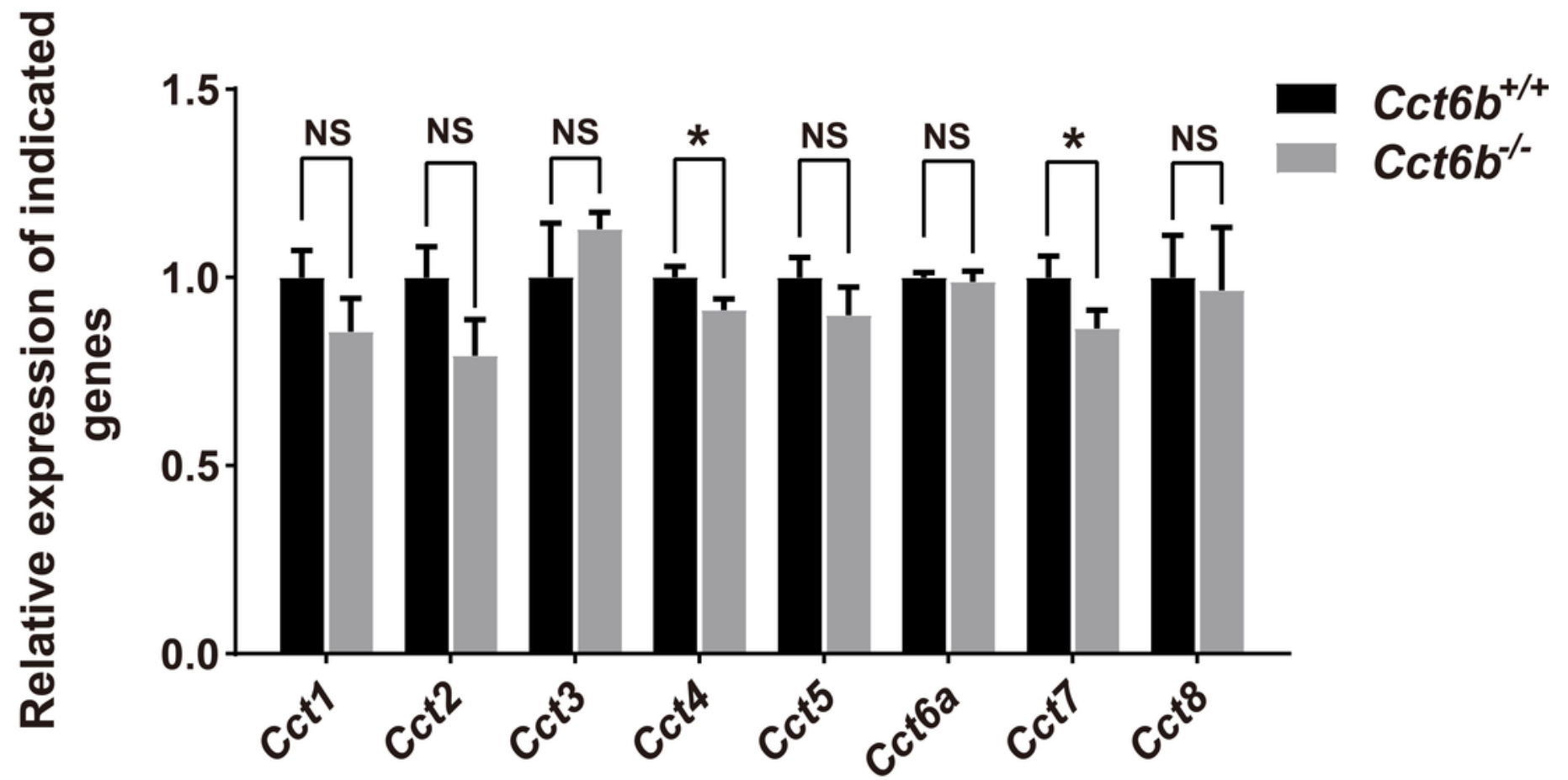

